\title{
Editorial
}

Nutritionat of
Metabolism

Published online: January 18, 2014

DOI: $\underline{10.1159 / 000357572}$

\section{Biomarkers in Growth}

\author{
Daniel J. Raiten ${ }^{a}$ Ramkripa Raghavan ${ }^{a}$ Klaus Kraemer ${ }^{b, c}$ \\ a Eunice Kennedy Shriver National Institute of Child Health and Human Development, National Institutes of Health, \\ Bethesda, Md., and ${ }^{\mathrm{b}}$ Johns Hopkins University, Bloomberg School of Public Health, Baltimore, Md., USA; \\ 'Sight and Life, Basel, Switzerland
}

\section{Key Words}

Biomarkers · Growth · Stunting · Biomarkers in growth

\begin{abstract}
Background: The current approaches to assess growth are limited to anthropometry, are insensitive and nonspecific, and do not enable an improved understanding of how nutrition might impact growth. Consequently, new tools to develop better standards of care and programs to address ongoing concerns about nutrition and health are needed. Methods: The Biomarkers of Nutrition for Development (BOND) project is designed to support the discovery, development, and use of current and new biomarkers of nutritional exposure, status, function, and effect. The Biomarkers in Growth (BIG) project was initiated as a BOND program to develop a roadmap for moving the nutrition and growth agenda forward. The first step in this project was a session jointly organized by the BOND Secretariat and Sight and Life at the 20th International Congress on Nutrition in Granada, Spain. Results: The BIG session outlined current approaches to evaluating growth and understanding of the role of nutrition in linear growth, body composition, and long-term health outcomes and the potential role of systems biology
\end{abstract}

in the assessment of the nutrition-growth relationship. Conclusion: The session presentations and deliberations highlighted the need for a concerted effort to address the critical gaps in our understanding of the biology and assessment of growth.

(c) 2014 S. Karger AG, Basel

\section{Introduction}

The proportion of overall disease burden attributable to childhood underweight has more than halved between 1990 and 2010; however, it remains the 8th highest risk worldwide. Moreover, among children under 5 years of age, childhood underweight was still the leading risk factor worldwide in 2010 [1]. Stunting and growth faltering remain significant concomitants of childhood undernutrition and continue to present complex global health challenges. According to Black et al. [2], an estimated 165 million children under 5 years of age are stunted and 52 million are wasted. Risk factors encompass poor nutrition including single/multiple micronutrient insufficiencies, low birth weight, inadequate breastfeeding, improper complementary feeding and caring practices, recurrent

\section{KARGER}

E-Mail karger@karger.com www.karger.com/anm
(C) 2014 S. Karger AG, Basel

0250-6807/14/0634-0293\$39.50/0
Daniel J. Raiten

6100 Executive Boulevard Bethesda, MD 20892 (USA)

E-Mail raitend@mail.nih.gov 
infections, and maternal nutrition/intrauterine growth restriction [3].

Although the magnitude of the problem is well characterized, the biology and our ability to ascertain either mechanisms or anything other than crude measures of remediation remain elusive. Further, the biology that distinguishes stunting, a chronic restriction of growth in height indicated by short stature (reduced height/age) from wasting, acute weight loss indicated by a low weight for height, and the role of nutrition in either have not been clearly delineated. Moreover, aside from the visible signs of poor linear growth or changes in body composition, many other short- (impacting numerous biological systems including brain/neurological development and impaired immune response/increased susceptibility to infection) and long-term (poor cognitive/behavioral/ work performance and increased susceptibility to chronic diseases including diabetes, cardiovascular risk, and obesity) adverse outcomes have been associated with early nutritional insults.

Programmatically, the issue of undernutrition is now further complicated by the alarming increase in maternal and young child overweight [4]. The dual burden of overand undernutrition has impacts on both individuals and populations. A need exists for better tools to describe the consequences and outcomes of these conditions as well as interventions to address them. There is a wide variety of tools that are available in resource-sufficient environments to assess growth but these are generally unattainable in resource-constrained settings and when dealing with large study populations. Although anthropometry offers benefits in terms of its relative ease of use, the availability of accepted standards for clinical and population use, and its lack of invasiveness, these measures are insensitive and nonspecific and only provide an 'early warning' with a decrease in linear growth velocity if carried out over time. In addition, they offer little to advance our understanding of those mechanisms to explain how nutrition might impact growth. The main drivers for better biomarkers of growth include:

- recognition of the need for and the impact of nutrition programs designed to prevent and ameliorate childhood over- and undernutrition

- a growing appreciation of the role of deficits in early growth (fetal, early infancy) as a major risk factor for the development of such adverse outcomes as chronic disease later in life, including type 2 diabetes and cardiovascular disease as well as impaired immune function and neurological development
- the need to gain a fuller understanding of the role of nutrition, broadly, and nutrients or nutrient clusters, specifially, within those biological systems involved in growth and related outcomes.

\section{Objectives}

To address these global health priorities, a concerted effort is required to support the discovery, development, validation, and implementation of current and new biomarkers that more clearly reflect the biology of growth and the role of nutrition therein. To begin the conversation and to develop a roadmap for moving this important agenda forward, the Biomarkers in Growth (BIG) project was conceptualized. The BIG project will be conducted under the aegis of the Biomarkers of Nutrition for Development (BOND) project housed at the Eunice Kennedy Shriver National Institute of Child Health and Human Development (NICHD), National Institutes of Health (NIH). The goals and objectives of the BOND project have been previously described [5].

As a first step in this project, a session was jointly organized by the BOND Secretariat and Sight and Life at the 20th International Congress on Nutrition in Granada, Spain. The aim of this session was to:

- discuss current approaches to evaluating growth and related outcomes

- outline the current understanding of the role of nutrition and specific nutrients in linear growth, body composition, and long-term health outcomes

- explore these relationships from a 'systems biology' perspective including endocrinology, immunology, and neurophysiology

- discuss growth within the context of other high-priority public health concerns (e.g. noncommunicable diseases and long-term health).

The session was chaired by Drs. Klaus Kraemer (Sight and Life) and Daniel Raiten (NICHD/NIH) and included a panel discussion following the content presentations that included Drs. Lindsay Allen (USDA), Andre Briend (University of Tampere Medical School), and Rosalind Gibson (University of Otago).

\section{Session Summary}

The session opened with a presentation by Zulfiqar Bhutta (Aga Khan University/SickKids Center for Global Child Health, Toronto) covering the current challenges 
with regard to growth in low- and middle-income countries. Dr. Bhutta outlined current data on the intransigent rates of stunting, the role of the first 1,000 days (i.e. pregnancy through the first 2 years of life), contributing environmental factors, limitations with regard to assessment tools, and how these factors impact the global efforts to more effectively progress in our quest to address this compelling public health problem.

Morey Haymond (Baylor College of Medicine) followed by providing an overview of the endocrinology of linear growth. Along with a brief overview of the biology, Dr. Haymond described the complex interplay between the myriad of factors (e.g. genetic/epigenetic, nutritional, environmental, and endocrine) that can influence linear growth. He highlighted the role of the intrauterine environment on both short- and long-term growth and noted that the timing of the nutritional insult(s) and relief from such factors are most likely critical and may ultimately influence the individual's final adult height. Because of its burgeoning role as a significant public health concern, Dr. Haymond described the impact of obesity and its metabolic correlates on growth. He emphasized the need for targeted studies collecting objective data which have a reasonable degree of validity using classical methods involving sequential measures of linear growth and their correlations with other biomarkers. He concluded with a brief overview of the currently available tools for assessing growth and reiterated the need for an expanded effort in this regard.

Prof. Alan Jackson (Southampton Biomedical Research Centre) provided an overview of the current understanding of the role of specific nutrients in growth. He opened his presentation with a discussion of several key aspects of linear growth including: the utility of current anthropometric measurements (e.g. length/height) and their reliably and validity, a brief discussion on the components of the bone matrix - collagen/cartilage and bone growth/turnover, mineralization and the role of calcium, vitamin $\mathrm{D}$, phosphorus, and other nutrients (e.g. magnesium, potassium, sodium, zinc, and copper), and a brief coverage of the impact of inflammation.

The major focus of Dr. Jackson's presentation was on conditionally essential nutrients, i.e. those that while produced endogenously may under certain circumstances be required in amounts beyond the body's capacity to meet its needs. He highlighted the particular importance of those amino acids involved in body composition and growth, emphasizing the particular importance of glutamine and related amino acids, arginine, proline, and the branched-chain amino acids (leucine, isoleucine, and va- line). He emphasized the underappreciated role of glycine $[6,7]$ and related amino acids (serine/cysteine/taurine: threonine, choline) in DNA, RNA, collagen, creatine, heme, and glutathione synthesis. In particular, he noted that collagen is composed of $30 \%$ glycine and $25 \%$ proline and that the metabolic demand for these conditionally essential amino acids can exceed the body's capacity to produce them.

Because of their importance, a need exists to integrate valid and reliable methods for assessing these amino acids as well as vigilance to ensure that when endogenous production is insufficient exogenous sources are available. Dr. Jackson cautioned for a balanced approach as, while a lower intake requires other sources, higher intakes can cause problems (e.g. the need to detoxify the excess). He shared data showing the beneficial effects of supplementation with glycine on growth in infants on low-protein diets and the utility of urinary concentrations of 5-L-oxoproline as a reliable biomarker of glycine status.

Dr. Jackson's 'take-home message' was that linear growth is dependent upon substrate availability for collagen formation. These processes are most likely to be limited by the availability of conditionally essential amino acids - prominently proline and glycine. This is especially likely to be problematic during periods when there are competitive demands (inflammation, infection, and xenobiotic challenge) $[8,9]$. He also emphasized the potential linkage between glycine (both in terms of its role in growth and as an excitatory and inhibitory neurotransmitter), growth, and cognitive outcomes [10].

The next presentation was offered by Ricardo Uauy who summarized some emerging data with regard to the impact of and conundrums raised by the dual burden of over- and undernutrition in children. He provided brief overviews of the global trends in childhood obesity and some of the results from the Growth and Obesity Chilean Cohort Study (GOCS). The GOCS is a longitudinal cohort study designed to assess the association of early growth and development of adiposity and metabolic risk [11]. The study evaluated 1,196 children who were eligible if they were 3.0-4.9 years of age, full-term singleton births, and not low birth weight (i.e. had a birth weight $\geq 2,500 \mathrm{~g}$ based on medical registry data) and had no physical or psychological conditions that could severely affect growth.

Dr. Uauy's summary of the GOCS study included the observation that the BMI at age 6 months is associated with height at age 7 years. He attributed this in part to a relation to accelerated skeletal maturation measured using ultrasound transmission at the wrist, i.e. faster bone 
maturation induced by excess adiposity. He also reported that children in the cohort with high BMI z-scores had a higher bone age than those with normal BMI z-scores. The BMI gain after 6 months of age was correlated with the achieved length at age 7 years; however, this effect disappeared after adjusting for bone age. His final point was that, based on the GOCS analyses, anthropometry alone will not suffice if the goal is to promote healthy growth and that assessment must include bone/hormonal maturation.

The final presentation was an exploration of the potential of a biological systems approach to nutrition by Ben van Ommen of TNO. He focused on the need to be more 'physiological' in our approach to nutrition, i.e. eschewing the traditional single-nutrient approach in favor of an approach that incorporates a view of nutrients within biological systems. Implicit in this approach is the recognition that: (1) nutrients do not operate in isolation and (2) there is an inherent flexibility in systems that allows the body to accommodate fluctuations in any component. On a molecular regulatory level, numerous processes are continually fine-tuning aspects of health, i.e. the phenotype, to maintain and regain homeostasis after dietary, metabolic, oxidative, inflammatory, and other challenges. Dr. van Ommen referred to this phenomenon as 'phenotypic flexibility' and characterized the interaction of multiple systems in this flexible manner as serving as a 'shock absorber' promoting health in the face of fluctuations in these systems as a consequence of a myriad of exposures (e.g. dietary, metabolic, genetic, inflammatory). Although a systems approach is complex in nature as it takes into account all relevant parameters involved in the process, a series of tools and examples have emerged that demonstrate its concrete applicability in health care. He described an application of this approach that has gained traction in the diagnosis and care of type 2 diabetes, where the complexity of the systems approach was taken into consideration while presenting a relatively simple solution to the health care arena, and outlined potential approaches by which systems biology may similarly be applied in the field of nutrition and the biomarker agenda.

\section{Panel Discussion}

Following the presentations, the guest panel responded with their thoughts about the materials offered and potential directions for the BIG project. Some of the most salient points included:
- Lindsay Allen reinforced the potential value of a systems biology approach to the nutrition/growth agenda.

- As with the application of this approach to the 'metabolic syndrome' offered by Dr. van Ommen, a systems biology approach to nutrition/growth will require building a systems model to predict differences in metabolic patterns between children growing normally and those who are not.

- Rosalind Gibson presented a model that highlights the synergism and interaction between an inadequate diet and infection in driving linear growth failure [12].

- While considering the role of nutrients in growth, it will be imperative to include the role of exposure (dietary intake and appetite) as a direct influence on nutrition within the context of this interactive model.

- In addition to the more well-characterized nutrients (e.g. vitamin $\mathrm{D}$, zinc, etc.), deficiencies of potassium and sodium impair lean tissue synthesis.

- Infection and inflammation are additional pathways through which growth is impacted, so it will be important to consider their contributions vis-à-vis the impact on digestion and absorption (e.g. elevated intestinal permeability, a reduced absorptive surface area and malabsoption), interactions with nutrients, and their impact on inflammatory responses.

- The importance of improving the indirect measurement tools of growth and body composition such as anthropometry, dual X-ray absorptiometry, and total body water via isotope dilution using deuterium and bioelectrical impedance were highlighted.

- Dr. André Briend emphasized the importance of stunting for the global health agenda and highlighted the need to more fully understand the links and potential mechanisms between stunting and neurological outcomes including cognitive development.

\section{Open Discussion}

The general discussion reinforced the importance of many of the issues addressed in the presentations as follows:

- The need to address the potential biological links between nutrition (essential and conditionally essential macro-/micronutrients), growth (normal and adverse outcomes such as stunting), and neurological function including cognitive and behavioral manifestations. With regard to the neurological aspects, it will be important to address these relationships from both a 
structural and a functional perspective as well as within the context of potential interactions with other biological systems (e.g. GI/brain, immune function/brain function, etc.).

- A clear priority is the need for a better understanding of the true nature/mechanisms of stunting, the role of nutrition, and how best to identify biomarkers that reflect those relationships.

- The need for better biomarkers reflecting the role of relevant biological systems (e.g. inflammation) and their impact on growth was highlighted. A related priority is the need for platforms, particularly those that could be utilized in resource-constrained settings, upon which markers reflecting nutrient status/function/effect and biomarkers of relevant systems (e.g. inflammation) were emphasized.

- A better understanding of the interactions between the biology of growth and external factors (social/behavioral) determinants is essential.

\section{Conclusions}

The content presented and the input from the discussions make it clear that a concerted effort is needed to address the myriad of issues related to an improvement in our understanding of the role of nutrition in growth and its concomitants. These materials will be valuable in informing the community and more specifically the BOND Secretariat about the best approaches for addressing these issues. The BIG project must be viewed within the context of the BOND mission of translation, service, and research. It is clear that the project will need to include a strong emphasis on systems biology. The nutrients to be considered in the BIG will be addressed as clusters within the relevant systems. As a next step, the BOND Secretariat will work with its partners and the BOND Steering Committee to begin forming the requisite expert panels to address the issues raised and the nutrients to be covered.

\section{Acknowledgements}

The authors thank Alexandra Porter for her help with the manuscript preparation.

The BIG session at the ICN was funded by Sight and Life.

\section{Disclosure Statement}

The authors declare no conflict of interest.

\section{References}

1 Lozano R, Naghavi M, Foreman K, Lim S, Shibuya K, Aboyans V, Abraham J, Adair T, Aggarwal R, et al: Global and regional mortality from 235 causes of death for 20 age groups in 1990 and 2010: a systematic analysis for the Global Burden of Disease Study 2010. Lancet 2012;380:2095-2128.

2 Black RE, Victora CG, Walker SP, Bhutta ZA, Christian P, de Onis M, Ezzati M, GranthamMcGregor S, Katz J, et al: Maternal and child undernutrition and overweight in low-income and middle-income countries. Lancet 2013;382:427-451.

-3 Christian P, Lee SE, Donahue Angel M, Adair LS, Arifeen SE, Ashorn P, Barros FC, Fall CH, Fawzi WW, et al: Risk of childhood undernutrition related to small-for-gestational age and preterm birth in low- and middle-income countries. Int J Epidemiol 2013;342:13401355.
4 Black RE, Victora CG, Walker SP, Bhutta ZA, Christian P, de Onis M, Ezzati M, GranthamMcGregor S, Katz J, et al: Maternal and child undernutrition and overweight in low-income and middle-income countries. Lancet 2013;382:427-451.

5 Raiten DJ, Namaste S, Brabin B, Combs G Jr, L'Abbe MR, Wasantwisut E, Darnton-Hill I: Executive summary - Biomarkers of Nutrition for Development: Building a Consensus. Am J Clin Nutr 2011;94:633S-650S.

6 Jackson AA: The glycine story. Eur J Clin Nutr 1991;45:59-65.

7 Harsing LG Jr, Matyus P: Mechanisms of glycine release, which build up synaptic and extrasynaptic glycine levels: the role of synaptic and non-synaptic glycine transporters. Brain Res Bull 2013;93:110-119.

8 Petrat F, Boengler K, Schulz R, de Groot H: Glycine, a simple physiological compound protecting by yet puzzling mechanism(s) against ischaemia-reperfusion injury: current knowledge. Br J Pharmacol 2012;165:20592072.
Knights KM, Sykes MJ, Miners JO: Amino acid conjugation: contribution to the metabolism and toxicity of xenobiotic carboxylic acids. Expert Opin Drug Metab Toxicol 2007;3: 159-168.

10 Carmans S, Hendriks JJ, Thewissen K, Van den Eynden J, Stinissen P, Rigo JM, Hellings $\mathrm{N}$ : The inhibitory neurotransmitter glycine modulates macrophage activity by activation of neutral amino acid transporters. J Neurosci Res 2010;88:2420-2430.

11 Corvalan C, Uauy R, Mericq V: Obesity is positively associated with dehydroepiandrosterone sulfate concentrations at $7 \mathrm{y}$ in Chilean children of normal birth weight. Am J Clin Nutr 2013;97:318-325.

12 Panter-Brick C, Lunn PG, Langford RM, Maharjan M, Manandhar DS: Pathways leading to early growth faltering: an investigation into the importance of mucosal damage and immunostimulation in different socio-economic groups in Nepal. Br J Nutr 2009;101:558567. 